\title{
Angeborene Immunität und Infektionen durch bekapselte Bakterien
}

\author{
Inborn Immunity and Infections by Capsuled Bacteria
}

Autoren

Institute
M. Knuf ${ }^{1,2}$, J. Faber $^{2}$

Klinik für Kinder und Jugendliche, Dr.-Horst-Schmidt-Kliniken $\mathrm{GmbH}$, Wiesbaden

2 Zentrum für Kinder- und Jugendmedizin, Universitätsmedizin Mainz

\section{Bibliografie}

Dol http://dx.doi.org/ 10.1055/s-0030-1256186

Online-Publikation: 14.3. 2011

Akt Dermatol 2011; 37:

159-163 @ Georg Thieme

Verlag KG Stuttgart · New York ISSN 0340-2541

\section{Korrespondenzadresse}

Prof. Dr. med. Markus Knuf Klinik für Kinder und Jugendliche Dr.-Horst-Schmidt-Kliniken Ludwig-Erhard-Str. 100 65199 Wiesbaden Markus.Knuf@

hsk-wiesbaden.de

\section{Zusammenfassung \\ $\nabla$}

Invasive Infektionen durch Meningokokken und Pneumokokken sind wichtige Ursachen für Morbidität und Letalität im Säuglings- und Kleinkindalter. Der klinische Verlauf variiert von relativ milden klinischen Manifestationen bis hin zu fulminantem septischem Schock mit einer Letalität bis zu $90 \%$. Immunologische Mechanismen, die von Geburt an aktiv sind (das sog. Angeborene Immunsystem), spielen eine wichtige Rolle bei der $\mathrm{Ab}$ wehr gegenüber invasiven Meningokokken- und Pneumokokken-Infektionen, insbesondere im „vulnerablen Fenster“ nach dem Abfall der müt-

Bekapselte Bakterien wie Neisseria meningitidis und Streptococcus pneumoniae sowie andere sind in der Kinder- und Jugendmedizin bedeutsame Erreger im Zusammenhang mit septischen Krankheitsbildern. Insbesondere Kleinkinder sind von Pneumokokken regelhaft besiedelt, wohingegen Meningokokken bei Adoleszenten und im jungen Erwachsenenalter besonders häufig vorkommen (๑ Tab. 1).

Invasive Erkrankungen durch Meningokokken hingegen, insbesondere durch die Serogruppe B und Serogruppe $C$, treten besonders häufig bei Säuglingen und Kleinkindern auf. Die Inzidenz für invasive Erkrankungen durch Neisseria meningitidis der Gruppe B betrug in den letzten Jahren etwa 10 je 100000 Einwohner [1] im 1. Lebensjahr ( Abb. 1). Absolut und für alle Serogruppen betrachtet erlitten 2007436 und 2008452 Menschen invasive Meningokokken-Erkrankungen [1]. 2007 waren 37 und 200844 Sterbefälle zu beklagen.

In Analogie zu den Meningokokken, ist die Inzidenz für invasive Pneumokokken-Erkrankungen im 1. Lebensjahr [2] deutlich höher als bei Schulkindern und Jugendlichen ( Tab.2). Obwohl Säuglinge und Kleinkinder überproportional mit terlich erworbenen Antikörper (Nestschutz) und vor der vollständigen Entwicklung adaptiver Immunmechanismen. Es wird zunehmend deutlich, dass Defekte in Schlüsselmolekülen des angeborenen Immunsystems, wie dem Mannose bindenden Lektin (MBL) oder Mitgliedern der Toll-likeRezeptor-Superfamilie (wie TLR4 und andere), für das Auftreten von Meningokokken- und/oder Pneumokokken-Infektionen disponieren, den klinischen Phänotyp und Verlauf modulieren oder beides. Dieser Effekt erscheint am meisten prävalent in der „Vulnerablen Phase“ des Säuglingsalters, bevor andere redundante Mechanismen des Immunsystems vollständig ausgereift sind.

Streptococcus pneumoniae und relevant mit Neisseria meningitidis besiedelt sind, erleiden absolut betrachtet relativ wenige Säuglinge und Kleinkinder invasive Erkrankungen. Hieraus resultieren folgende Fragen:

1. Warum sind gerade Säuglinge und Kleinkinder prominent betroffen?

2. Wenn es für die Altersverteilung eine spezifische Ursache gibt, warum erkranken dann nicht mehr Kinder?

Eine zentrale Rolle in der Auseinandersetzung zwischen dem „Wirt“ und dem bekapselten Bakterium spielt bei Säuglingen und Kleinkindern die angeborene Immunität („innate immunity“). Unmittelbar nach der Entbindung sind Neugeborene durch diaplazentar übertragene, schützende, maternale Antikörper zunächst gegen invasive Erkrankungen durch Meningokokken (oder Pneumokokken) geschützt [3]. In dem „Vulnerablen“ Zeitraum nach dem 6 . Lebensmonat sind transplazentar erworbene, mütterliche Antikörperspiegel abgefallen und erworbene Mechanismen der adaptiven Immunität noch nicht vollständig entwickelt. Daher spielen in dieser Phase angeborene Immunmechanismen, welche von Geburt an konstitutiv aktiv sind, eine zentrale Rolle in der 
Tab. 1 Besiedlungsraten von Neisseria meningitidis und Streptococcus pneumoniae in Abhängigkeit von Altersgruppe.

\begin{tabular}{|c|c|}
\hline \multicolumn{2}{|l|}{ Neisseria meningitidis* } \\
\hline \multicolumn{2}{|l|}{ Altersgruppe } \\
\hline Kindergarten/Grundschule & $2 \%$ \\
\hline Haupt-/Realschule & $7,6 \%$ \\
\hline Gymnasialoberstufe & $17,9 \%$ \\
\hline Bundeswehrrekruten & $32,6 \%$ \\
\hline \multicolumn{2}{|l|}{ Streptococcus pneumoniae ${ }^{* *}$} \\
\hline \multicolumn{2}{|l|}{ Altersgruppe } \\
\hline Kinder unter 6 Jahren & bis zu $60 \%$ \\
\hline Grundschulkinder & $35 \%$ \\
\hline Kinder über 10 Jahre & $25 \%$ \\
\hline Erwachsene mit Kindern im Haushalt & $18-29 \%$ \\
\hline Erwachsene ohne Kinder im Haushalt & $6 \%$ \\
\hline \multicolumn{2}{|c|}{$\begin{array}{l}\text { Quellen: * Meningokokkenträgerstudie Winter 1999/2000. Institut für } \\
\text { Hygiene- und Mikrobiologie der Universität Würzburg. } \\
\text { ** Black S, Shinefield H. Issues and challenges: pneumococcal vaccination in } \\
\text { pediatrics. Pediatr Ann 1997; } 26: 355-360\end{array}$} \\
\hline
\end{tabular}

Immunabwehr invasiver Infektionen durch bekapselte Erreger $[4,5]$. In dieser frühen antikörperunabhängigen Immunantwort gegen z.B. Meningokokken-Infektionen spielt das Komplementsystem eine wichtige Rolle. Neben der Aktivierung des Komplementsystems durch spezifische Antikörper über den sogenannten klassischen Aktivierungsweg, sind in dieser „vulnerablen“ Phase zwei von Antikörpern unabhängige Wege des angeborenen Immunsystems fähig, das Komplementsystem zu aktivieren $(\bullet$ Abb. 2). Hierbei spielt der sogenannte „alternative Weg der Komplementaktivierung“" mittels Bildung der sogenannten C3Konvertase und Aktivierung der späteren Komplementkomponenten und des „Membran-Angriff-Komplexes“ eine wichtige Rolle. Die Bedeutung eines Defektes in den antikörperunabhängigen Wegen der Komplementaktivierung wird unterstrichen durch die Tatsache, dass sowohl Individuen mit genetischen Defekten im Properdin kodierenden CFP-Gen als auch Patienten mit
Tab. 2 Altersverteilung invasiver Pneumokokken-Erkrankungen [2].

\begin{tabular}{llc} 
Alter & $\begin{array}{l}\text { Invasive Pneumo- } \\
\text { kokken-Erkrankungen } \\
\text { pro 10000/Jahr }\end{array}$ & $\begin{array}{l}\text { Meningitiden } \\
\text { pro 100 000/Jahr }\end{array}$ \\
\hline 0 bis 5 Monate & 17,3 & 8,5 \\
\hline 6 bis 11 Monate & 29,4 & 12,6 \\
\hline 1 Jahr & 16,3 & 4,6 \\
\hline 2 bis 4 Jahre & 5,4 & 1,8 \\
\hline 5 bis 15 Jahre & 1,1 & 0,4 \\
\hline < 5 Jahre & $\mathbf{1 1 , 1}$ & $\mathbf{4 , 1}$ \\
\hline
\end{tabular}

hereditärem Faktor-D-Mangel ein erhöhtes Risiko für invasive Meningokokken-Infektionen aufweisen [6,7].

Neben dem sogenannten alternativen Weg kann im Rahmen der „innate Immunity“ eine von Antikörpern unabhängige Aktivierung des Komplementsystems über das Mannose bindende Lektin (MBL) mit Aktivierung des sogenannten Lektins erfolgen [8]. MBL ist ein gut definierter, löslicher Mustererkennungsrezeptor und ein wichtiger Bestandteil des angeborenen Immunsystems. In die Blutbahn eingedrungene Meningokokken werden an MBL gebunden, was zu einer Aktivierung der MBL-assoziierten SerinProtease 1 sowie der MBL-assoziierten Serin-Protease 2 führt $[9,10]$. Im weiteren Verlauf folgt die Aktivierung der späten Komplementkomponenten und Bildung des „Membran-Angriff-Komplexes“. Neben der Aktivierung des Komplementsystems über den Lektin-Aktivierungsweg agiert MBL auch als Opsonin durch Bindung von Proteinen auf der äußeren Zellmembran von Neisseria meningitidis und erleichtert so eine antikörperunabhängige Opsonophagozytose [11]. In den vergangenen Jahren wurden im humanen MBL-Gen mehrere strukturelle Varianten der kodierenden Gen-Region sowie im Promotorbereich identifiziert, die im Vergleich zum Wildtyp-Allel mit einer erniedrigten MBL-Plasmakonzentration einhergehen und mit einer signifikant erhöhten Anfälligkeit gegenüber invasiven Meningokokken-Infektionen assoziiert sind $[12,13]$.

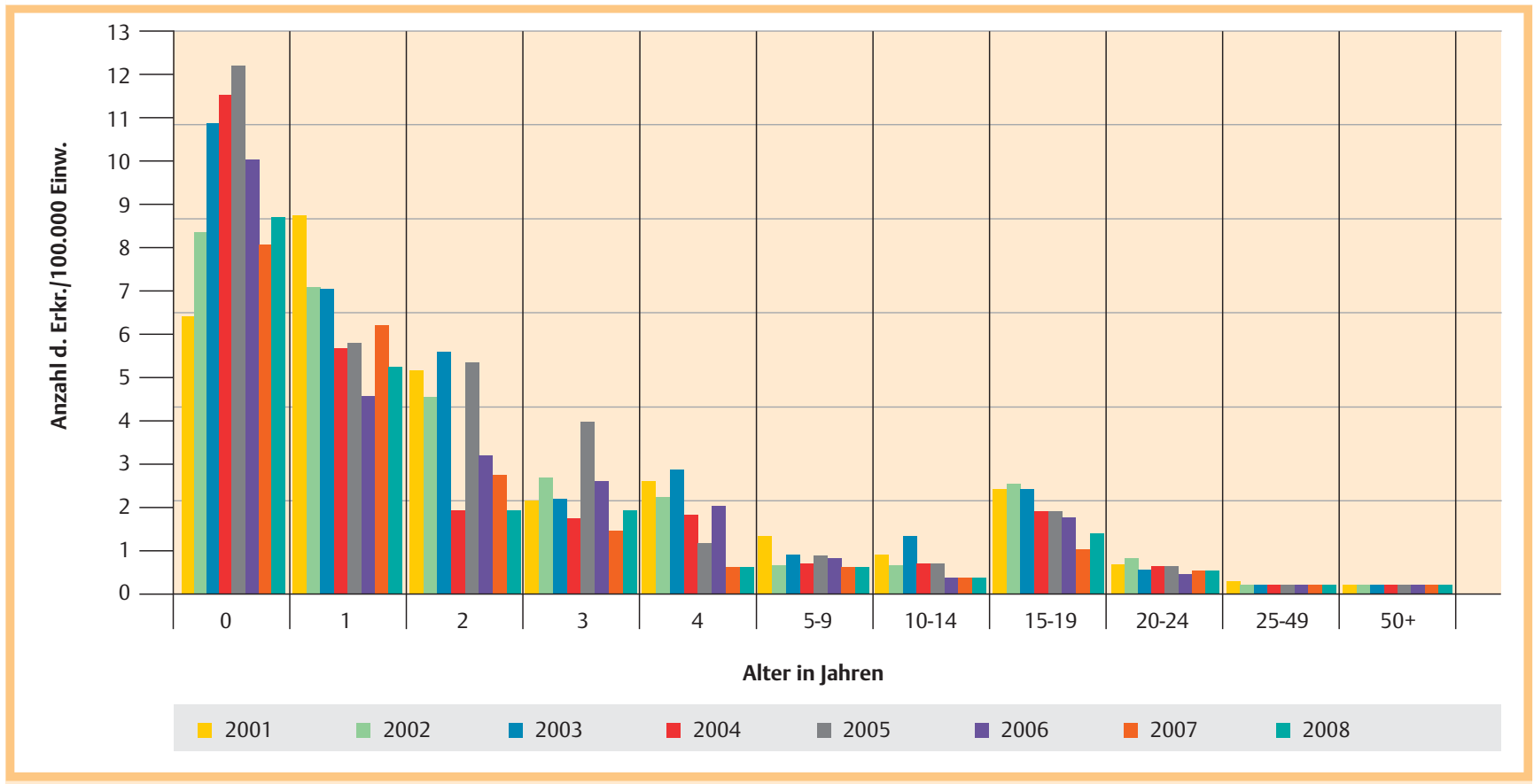

Abb. 1 Invasive Erkrankungen durch Meningokokken der Serogruppe B nach Alter, IfSG-Meldedaten (hochgerechnet), Deutschland 2001 - 2008 [1]. 


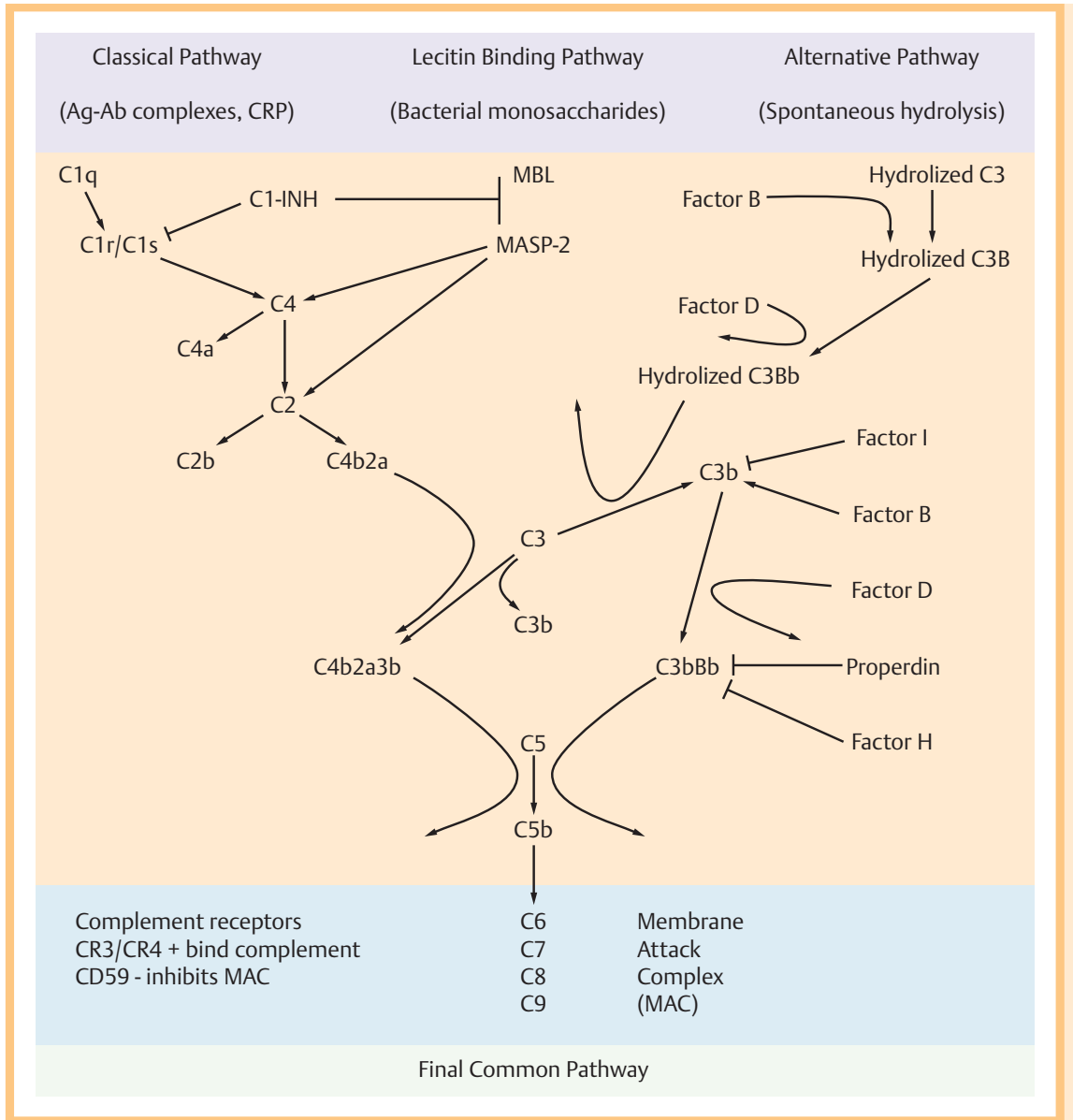

Abb. 2 Das Komplementsystem (Quelle: Pettigrew $\mathrm{H}$ et al. Clinical significante of complement deficiencies. Ann N Y Acad Sci 2009; 108: 1173). Abkürzungen/Übersetzungen: Classical pathway $=$ Klassischer Weg, Lectin Binding Pathway $=$ Lektin-Aktivierungsweg, Alternative Pathway $=$ Alternativer $\mathrm{Weg},(\mathrm{Ag}-\mathrm{Ab}$ complexes, CRP) = (Antigen-Antikörper-Komplexe, CRP), (Bacterial monosaccharides $)=($ Bakterielle Monosaccharide $)$, (Spontaneous hydrolysis) = (Spontane Hydrolyse), Hydrolized $=$ hydrolysiertes, C1-INH = C1-Inhibitor, MAC $=$ Membran-Angriffs-Komplex, MASP2 = Mannose bindende, Lektin-assoziierte Serinprotease, $\mathrm{MBL}=$ Mannose bindendes Lektin, $\mathrm{CRP}=\mathrm{C}$-reaktives Protein, Complement receptors $=$ KomplementRezeptoren, binds = bindet, inhibits = inhibiert, Final common pathway = Gemeinsamer Reaktionsweg.

Tab. 3 Häufigkeitsverteilung von MBL-Exon-1-Varianten [13].

\begin{tabular}{|c|c|c|c|c|c|}
\hline \multirow[t]{2}{*}{ Gruppe } & \multicolumn{3}{|l|}{$\begin{array}{l}\text { Geno- } \\
\text { typ n } \\
(\%)\end{array}$} & \multirow[t]{2}{*}{$\begin{array}{l}\text { 0-Allel- } \\
\text { frequenz } \\
(\%)\end{array}$} & \multirow[t]{2}{*}{$p=$} \\
\hline & $A / A$ & $\mathrm{~A} / 0$ & $0 / 0$ & & \\
\hline Gesamt & $\begin{array}{c}60 \\
(68,2)\end{array}$ & $\begin{array}{l}25 \\
(28,4)\end{array}$ & $\begin{array}{l}3 \\
(3,4)\end{array}$ & 17,6 & $<0,001$ \\
\hline $\begin{array}{l}\text { Patienten } \\
<2 \text { LJ. }\end{array}$ & $\begin{array}{c}17 \\
(60,7)\end{array}$ & $\begin{array}{l}11 \\
(39,3)\end{array}$ & $0(0)$ & 19,6 & $<0,001$ \\
\hline $\begin{array}{l}\text { Patienten } \\
<1 \text {. LJ }\end{array}$ & $\begin{array}{c}6 \\
(42,9)\end{array}$ & $\begin{array}{c}8 \\
(57,1)\end{array}$ & $0(0)$ & 28,5 & $<0,001$ \\
\hline Kontrolle & $\begin{array}{l}101 \\
(91,8)\end{array}$ & $\begin{array}{c}9 \\
(8,2)\end{array}$ & $0(0)$ & 4,1 & - \\
\hline
\end{tabular}

In $\odot$ Tab. 3 wird die Häufigkeitsverteilung von MBL-Exon-1-Varianten in einem Kollektiv von Säuglingen und Kleinkindern mit invasiven Meningokokken-Infektionen im Vergleich zu einer gesunden Kontrollpopulation zusammengefasst.

Dabei konnte der stärkste Einfluss eines MBL-Mangels als Risikofaktor für eine invasive Meningokokken-Infektion in dieser Altergruppe nachgewiesen werden [13].

\section{Bedeutung der Toll-like-Rezeptor-Proteinfamilie}

Lipopolysaccharid (LPS) ist eine Haupt-Glycolipid-Komponente der äußeren Membran von Meningokokken und anderer gramnegativer Bakterien. Kürzlich konnten die molekularen Mecha-
Tab. 4 Genotyp-Verteilung der Asp299Gly-Mutation bei Patienten mit Meningokokken-Infektion und gesunden Kontrollprobanden ( ${ }^{*}$ signifikant, exakter Fischer-Test [2-seitig]) [19].

\begin{tabular}{llllll} 
Kollektiv & $\begin{array}{l}\text { Geno- } \\
\text { typ n } \\
\text { (\%) }\end{array}$ & & & $\begin{array}{l}\text { Allel } \\
\text { 299Gly }\end{array}$ & $\boldsymbol{p}=$ \\
& Asp/ & Asp/ & Glyl & & \\
& Asp & Gly & Gly & & \\
IMK, & 165 & 27 & 5 & $9,4 \%$ & 0,247 \\
gesamt & $(83,7)$ & $(13,7)$ & $(2,5)$ & & \\
\hline IMK, & 26 & 9 & 2 & $17,6 \%$ & $0,008^{*}$ \\
Alter $<1$ J & $(70,2)$ & $(24,3)$ & $(5,4)$ & & \\
\hline $\begin{array}{l}\text { Kontroll- } \\
\text { gruppe }\end{array}$ & 96 & 13 & $0(0)$ & $6,0 \%$ & \\
\hline
\end{tabular}

nismen, mit denen das angeborene Immunsystem LPS erkennt, genauer beschrieben werden. Der Toll-like-Rezeptor 4 (TLR4) wurde als wichtigster Erkennungsrezeptor des angeborenen Immunsystems identifiziert $[14,15]$. Die Bindung von LPS an einen Komplex, der aus TLR4 und dem Ko-Molekül MD-2 besteht, führt zur Stimulation einer intrazellulär lokalisierten Signaltransduktionskaskade mit konsekutiver Aktivierung von weiteren sogenannten Adaptermolekülen z.B. MyD88 [16], TRIF, TIRAP und TRAM [17]. Folge dieser Aktivierung ist die Ausschüttung proinflammatorischer Zytokine, wie z.B. Tumornekrosefaktor- $\alpha$ (TNF- $\alpha$ ), IL-1, IL-6, IL-12 und anderer. Nachdem Daten aus dem Mausmodell vorlagen, die darauf hinwiesen, dass TLR4-Defekte eine bedeutsame Rolle in der Pathogenese invasiver Meningo- 


\begin{tabular}{|c|c|c|c|c|c|c|c|}
\hline & & Alle Patiente & & & Patienten $<2$ & hre & \\
\hline & & Auftreten (\% & Anzahl/Gesam & & Auftreten (\% & Anzahl/Gesan & \\
\hline & & $\begin{array}{l}\text { 299Asp/Asp } \\
n=165\end{array}$ & $\begin{array}{l}\text { 299Asp/Gly } \\
n=27\end{array}$ & p-Wert & $\begin{array}{l}\text { 299Asp/Asp } \\
n=61\end{array}$ & $\begin{array}{l}\text { 299Asp/Gly } \\
\mathrm{n}=12\end{array}$ & p-Wer \\
\hline $\begin{array}{l}\text { Fataler } \\
\text { Verlauf }\end{array}$ & $\mathrm{Ja}$ & $\begin{array}{l}68,4 \\
(13 / 19)\end{array}$ & $\begin{array}{l}31,6 \\
(6 / 19)\end{array}$ & 0,021 & $\begin{array}{l}57,1 \\
(8 / 14)\end{array}$ & $\begin{array}{l}42,8 \\
(6 / 14)\end{array}$ & 0,006 \\
\hline & Nein & $\begin{array}{l}87,9 \\
(152 / 173)\end{array}$ & $\begin{array}{l}12,1 \\
(21 / 173)\end{array}$ & & $\begin{array}{l}89,8 \\
(53 / 59)\end{array}$ & $\begin{array}{l}10,2 \\
(6 / 59)\end{array}$ & \\
\hline Ventilation & $\mathrm{Ja}$ & $\begin{array}{l}81,8 \\
(45 / 55)\end{array}$ & $\begin{array}{l}18.1 \\
(10 / 55)\end{array}$ & 0,298 & $\begin{array}{l}62,5 \\
(15 / 24)\end{array}$ & $\begin{array}{l}37,5 \\
(9 / 24)\end{array}$ & 0,002 \\
\hline & Nein & $\begin{array}{l}87,6 \\
(120 / 137)\end{array}$ & $\begin{array}{l}12,4 \\
(17 / 137)\end{array}$ & & $\begin{array}{l}93,8 \\
(46 / 49)\end{array}$ & $\begin{array}{c}6,2 \\
(3 / 49)\end{array}$ & \\
\hline Inotropika & $\mathrm{Ja}$ & $\begin{array}{l}84,3 \\
(70 / 83)\end{array}$ & $\begin{array}{l}15,6 \\
(13 / 83)\end{array}$ & 0,579 & $\begin{array}{l}75 \\
(24 / 32)\end{array}$ & $\begin{array}{l}25 \\
(8 / 32)\end{array}$ & 0,081 \\
\hline & Nein & $\begin{array}{l}87,2 \\
(95 / 109)\end{array}$ & $\begin{array}{l}12,8 \\
(14 / 109)\end{array}$ & & $\begin{array}{l}90,2 \\
(37 / 41)\end{array}$ & $\begin{array}{c}9,8 \\
(4 / 41)\end{array}$ & \\
\hline
\end{tabular}

Tab. 5 Frequenz von TLR4 299Asp/Asp-Wild-Typ und heterozygoten TLR4 299Asp/ Gly-Genotypvarianten in Assoziation mit klinischen Parametern bei Kindern mit invasiven MeningokokkenInfektionen [20].
kokken-Infektionen spielen, konnten nunmehr auch Defekte im humanen TLR4-Rezeptor als Risikofaktor für das Auftreten invasiver Meningokokken-Infektionen identifiziert werden [18,19].

- Tab. 4 fasst die Häufigkeitsverteilung von heterozygoten und homozygoten Mutationen bei Patienten mit invasiven Meningokokken-Erkrankungen zusammen. Besonders häufig und signifikant sind Säuglinge zu beobachten gewesen, die bei homozygoter und heterozygoter Mutationslage an einer invasiven Meningokokken-Infektion erkrankten.

Im Vergleich zu älteren Patienten ist der klinische Verlauf [20] bei den unter 1-Jährigen besonders ungünstig ( Tab. 5). Die vorliegenden Daten deuten darauf hin, dass Defekte im TLR4-Signaltransduktionsweg einen gewichtigen Risikofaktor für einen schweren klinischen Verlauf invasiver Meningokokken-Infektionen und anderer gramnegativer Infektionen darstellen.

TLR4-Mutationen stellen einen Risikofaktor dar, es ist jedoch auch wahrscheinlich, dass andere genetische Defekte des angeborenen Immunsystems, möglicherweise im Zusammenspiel mit TLR4-Mutationen, als Ko-Faktoren für das Auftreten invasiver Meningokokken-Infektionen agieren. Eine mögliche Erklärung für die Abnahme der invasiven Infektionen im voranschreitenden Alter ist das Vorliegen von redundanten, adaptativen Mechanismen, die die Effekte der Mutation im angeborenen Immunsystem kompensieren. Eine bedeutende Rolle bei der Entwicklung invasiver Pneumokokken-Erkrankungen spielen ebenfalls Mutationen im sogenannten Toll-like-Rezeptor-System. Mutationen bei den Toll-like-Rezeptoren 2, 4 und 9 konnten tierexperimentell als Risikofaktoren für den besonders schweren schlechten Verlauf einer Pneumokokken-Infektion identifiziert werden [21].

Zusammenfassend kann festgestellt werden, dass Defekte der angeborenen Immunität in der vulnerablen Phase vor Ausreifung des adaptiven Immunsystems phänotypisch evident werden. Mechanismen des Immunsystems sind auch bei Säuglingen redundant bzw. multifaktoriell angelegt. Daher folgt eine Teilkompensation und nur im „ungünstigsten Fall“ die Ausbildung einer invasiven Erkrankung. Jenseits des 1. Lebensjahres ist eine Kompensation von „Defekten“ der angeborenen Immunität durch adaptative Immunmechanismen zu erwarten.

\section{Abstract}

\section{Inborn Immunity and Infections by Capsuled Bacteria}

Meningococcal and pneumococcal diseases are important causes of morbidity and mortality in infancy and childhood. The clinical course ranges from relatively benign clinical manifestations to fulminant septic shock with mortality rate up to $90 \%$. Immune components, which are constitutively present from birth (the innate immune system) help to protect infants from meningococcal and pneumococcal infection especially in the „vulnerable window“ after decline of transplacental acquired maternal antibodies and before full development of adaptive immune mechanisms. Accumulating evidence suggests that genetic defects in key components of the innate immune system such as Mannose binding Lectin or members of the Toll like receptor Superfamily (TLR4 and others) might either predispose for the onset of meningococcal or pneumococcal disease or modulate the clinical phenotype or both. This effect seems to be most predominant in the vulnerable phase of infancy before other redundant immune mechanisms are not yet fully developed.

\section{Literatur}

1 Epidemiologisches Bulletin, Nr. 45, 2009

2 Epidemiologisches Bulletin, Nr. 31, 2006

3 Knuf M, Hrsg. Invasive Meningokokken-Infektionen. Bremen: UNIMED; 2009: 34

4 Schneider MC et al. Interactions between Neisseria meningitidis and the complement system. Trends Microbiol 2007; 15: 233-240

5 van Deuren M, Brandtzaeg P, van der Meer JW. Update on meningococcal disease with emphasis on pathogenesis and clinical management. Clin Microbiol Rev 2000; 13: 44-166

6 Fijen CA et al. Assessment of complement deficiency in patients with meningococcal disease in The Netherlands. Clin Infect Dis 1999; 28: $98-105$

7 Sprong $T$ et al. Deficient alternative complement pathway activation due to factor D deficiency by 2 novel mutations in the complement factor D gene in a family with meningococcal infections. Blood 2006; 107: $4865-4870$

8 Kuhlman M, Joiner K, Ezekowitz RA. The human mannose-binding protein functions as an opsonin. J Exp Med 1989; 169: 1733-1745

9 Thiel $S$ et al. A second serine protease associated with mannose-binding lectin that activates complement. Nature 1997; 386: 506 - 510

10 Matsushita M, Fujita T. Activation of the classical complement pathway by mannose-binding protein in association with a novel C1s-like serine protease. J Exp Med 1992; 176: 1497-1502 
11 Jack DL et al. Mannose-binding lectin enhances phagocytosis and killing Neisseria meningitides by human macrophages. J Leukoc Biol 2005; 77: $328-336$

12 Peterslund NA et al. Association between deficiency of mannose-binding lectin and severe infections after chemotherapy. Lancet 2001; 358 : $637-638$

13 Faber $J$ et al. Age-dependent association of human mannose-binding lectin mutations with susceptibility to invasive meningococcal disease in childhood. Pediatr Infect Dis J 2007; 26: 243 - 246

14 Medzhitov R, Preston-Hurlburt CA, Janeway A. A human homologue of the Drosophila Toll protein signals activation of adaptive immunity. Nature 1997; 388: 394-397

15 Wright SD. Toll, a new piece in the puzzle of innate immunity. J Exp Med 1999; 189: 605-609

16 Kawai $T$ et al. Unresponsiveness of MyD88-deficient mice to endotoxin. Immunity 1999; 11: 115-122
17 Yamamoto $M$ et al. TRAM is specifically involved in the Toll-like receptor 4-mediated MyD88-independent signalling pathway. Nat Immunol 2003; 4: 1144-1150

18 Smirnova I et al. Assay of locus-specific genetic load implicates rare Toll-like receptor 4 mutations in meningococcal susceptibility. Proc Natl Acad Sci USA 2006; 100: 6075-6080

19 Faber J et al. Human Toll-like receptor 4 mutations are associated with susceptibility to invasive meningococcal disease in infancy. Pediatr Infect Dis J 2006; 25: 80-81

20 Faber J et al. A Toll-like receptor 4 variant is associated with fatal outcome in children with meningococcal disease in infancy. Acta Pediatr 2009; 98: 548-552

21 Klein $M$ et al. Innate immunity to pneumococcal infection of the central nervous system depends on toll-like receptor (TLR) 2 and TLR4. JID 2008; 198: 1028 